\title{
Odontología bajo anestesia general en el paciente con epilepsia: Reporte de caso y revisión de literatura
}

\author{
Dentistry under general anesthesia in the patient with epilepsy. Case report and literature review
}

Ana Cristina López Torres ${ }^{1, a ; 2 b ; 3 c}$, Carlos Eduardo Alfaro Pacheco ${ }^{\text {2,b, f; } 4 \text {, }}$, Lillie Elizabeth Abanto Silva ${ }^{2, b, d, f}$

\begin{abstract}
RESUMEN
La atención clínica del paciente portador de epilepsia (EP) asociada a discapacidad intelectual (DI), representa un reto para el estomatólogo por su difícil manejo conductual. Estos pacientes requerirán de alternativas para poder llevar a cabo un tratamiento odontológico exitoso. La anestesia general (AG), es una alternativa ante la falla de las técnicas de manejo conductual, existencia de compromiso sistémico que lo amerite, acceso médico especializado distante al lugar de residencia o por circunstancias particulares de cada caso. Se presenta el caso de un paciente varón de 27 años, con diagnóstico de EP asociada a DI, de difícil manejo conductual atendido bajo anestesia general en el servicio de Estomatología de Pacientes Especiales de la Universidad Peruana Cayetano Heredia. Objetivo: Este artículo busca presentar una revisión de la literatura y el Tratamiento Odontológico Integral bajo anestesia general de un paciente con Epilepsia asociada a Discapacidad Intelectual moderada. Conclusiones: Los pacientes con estas patologías de fondo son propensos a presentar deterioro del órgano bucal, por lo que requerirán prestaciones estomatológicas constantes. Por la poca colaboración de este tipo de pacientes, se debe considerar a la AG como alternativa para brindar el el tratamiento odontológico integral en un solo acto operatorio.

PALABRAS CLAVE: Atención dental para personas con discapacidades, discapacidad intelectual, epilepsia, anestesia general .
\end{abstract}

\section{SUMMARY}

The clinical care of patients with epilepsy (EP) associated with intellectual disability (ID) represents a challenge for the stomatologist because of its difficult behavioral management. These patients will require alternatives to carry out a successful dental treatment. General anesthesia (AG), is an alternative to the failure of behavioral management techniques, existence of systemic commitment that warrants it, specialized medical access distant from the place of residence or due to particular circumstances of each case. We present the case of a 27-yearold male patient, with a diagnosis of EP associated with ID, of difficult behavioral management treated under general anesthesia in the Special Patients Stomatology service of the Peruvian University Cayetano Heredia. Objective: This article seeks to present a review of the literature and Comprehensive Dental Treatment under general anesthesia of a patient with Epilepsy associated with moderate Intellectual Disability. Conclusions: Patients with these underlying pathologies are prone to present deterioration of the oral organ, so they will require constant stomatological benefits. Due to the low collaboration of this type of patients, the AG should be considered as an alternative to provide the TOI in a single operative act.

KEY WORDS: Dental care for people with disabilities, intellectual disability, epilepsy, general anesthesia.

Facultad de Odontología, Universidad de Costa Rica. San José, Costa Rica

Facultad de Estomatología, Universidad Peruana Cayetano Heredia. Lima, Perú.

Facultad de Educación, Universidad Peruana Cayetano Heredia. Lima, Perú.

Facultad de Medicina, Universidad Peruana Cayetano Heredia. Lima, Perú.

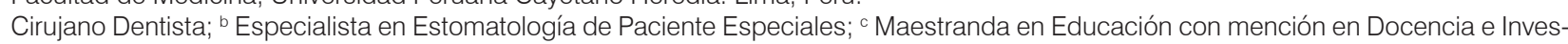
tigación en Educación Superior; ' Maestranda en Estomatología con mención en Estomatología de Pacientes Especiales; ${ }^{e}$ Maestrando en Geriatría y Gerontología; ' Docente de la Especialidad Estomatología de Paciente Especiales 


\section{INTRODUCCIÓN}

Los trastornos del neurodesarrollo incluyen: Discapacidad intelectual(DI), epilepsia (EP), trastornos del espectro autista (TEA) y parálisis cerebral infantil (PCI) $(1,2)$. La EP está relacionada como el causal del daño cerebral en pacientes con DI, entre $35 \%$ a $40 \%$ de niños con EP también tienen DI. La gravedad de la DI está directamente relacionada con la frecuencia y severidad de las crisis epilépticas. Es por ello, que la EP afecta alrededor del $20 \%$ en personas con DI leve y puede llegar a afectar hasta $50 \%$ de los pacientes con DI de severa a profunda $(3,4)$.

El término "epilepsia" engloba a un conjunto de trastornos neurológicos caracterizados por procesos recurrentes y crónicos de actividad paroxística de las funciones neuronales llamadas crisis epilépticas (CE). Las CE que corresponden a descargas eléctricas anómalas, excesivas e incontroladas de un gran número de neuronas. La clínica es heterogénea, desde alteraciones motoras, de la conciencia (con y sin manifestaciones motoras), fenómenos sensoriales o psíquicos y/o convulsiones. La EP afecta del 0,5 a 2\% de la población mundial, siendo de mayor prevalencia en países en vías de desarrollo (3,5-7).

Los defectos cerebrales congénitos, factores genéticos, complicaciones natales, infecciones y traumatismos son las causas etiológicas de la EP en personas menores de 30 años de edad; mientras que en mayores de 50 años la enfermedad cerebrovascular y tumores son las causantes de EP para este grupo poblacional (8). La clasificación internacional de las CE de 2017 está basada en las características semiológicas y estudios con electroencefalografía. En el primer nivel, las CE se clasifican en crisis de inicio focal, de inicio generalizado o de inicio desconocido y el segundo nivel menciona los tipos de crisis (figura 1) $(7,9,10)$.

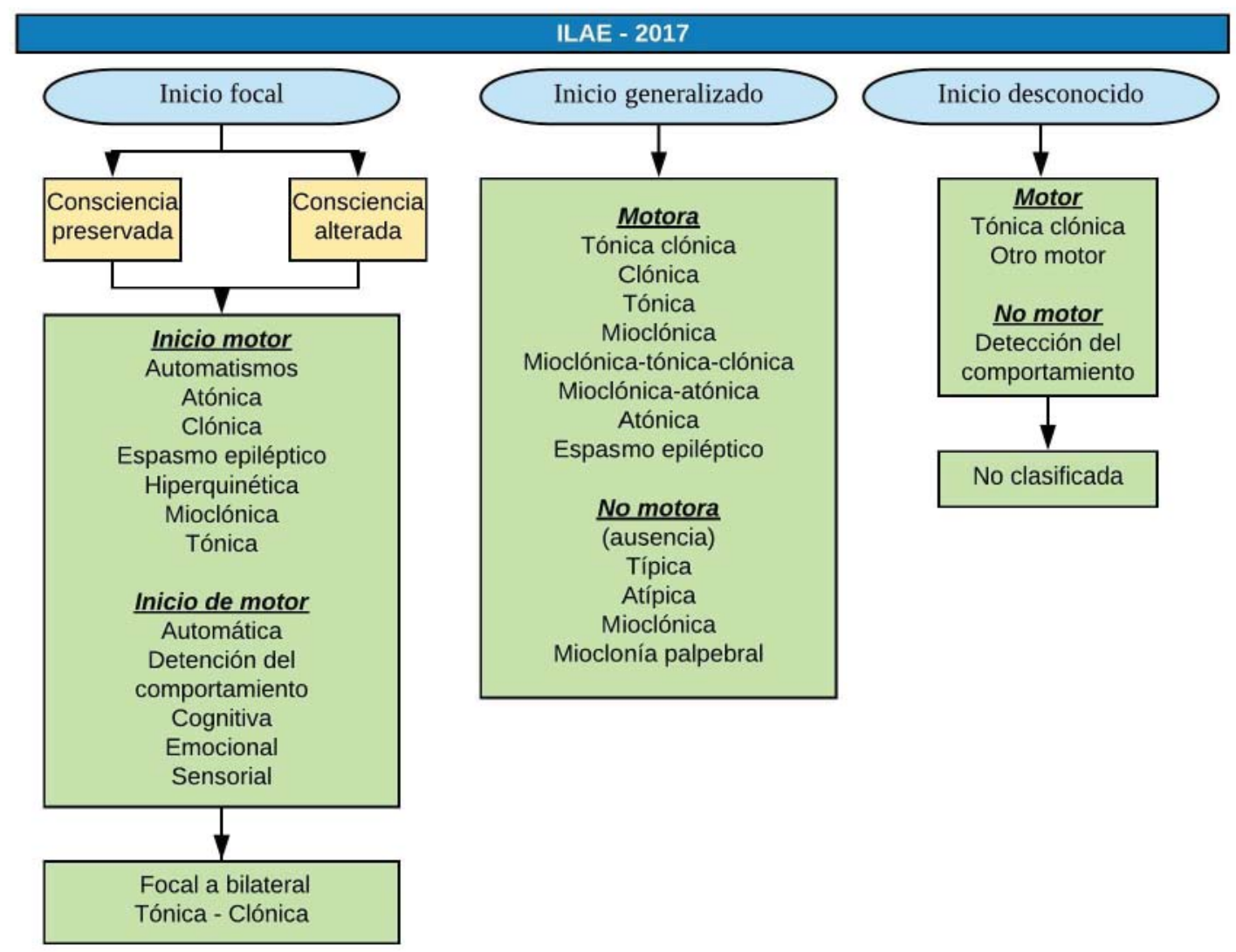

Figura 1. Clasificación operacional extendida de los tipos de crisis, ILAE - 2017 (9). 
La DI asociada a EP, representa un reto para el estomatólogo en la atención ambulatoria por el difícil manejo conductual. El deterioro de la salud oral del paciente con EP se asocia a una limitada destreza en la técnica de cepillado, poca colaboración y/o desconocimiento del cuidador en la preservación de la salud oral $(3,11)$. Cabe señalar que los medicamentos anticonvulsivantes tienen efectos secundarios sobre la mucosa oral y, por otro lado, las fracturas dentoalveolares secundarias a caídas durante las CE son frecuentes $(3,11,12)$. Bajo estas circunstancias, es necesario recurrir a otras alternativas para poder llevar a cabo el tratamiento odontológico con éxito, debiendo ser la anestesia general (AG) una alternativa ante la falla de las técnicas de manejo conductual, compromiso sistémico importante, el acceso médico especializado distante al lugar de residencia y/o circunstancias particulares de cada caso; exigiendo así un enfoque biopsicosocial de la práctica odontológica. Trabajar bajo AG permite plantear tratamientos odontológicos de forma integral (TOI) y en un solo acto operatorio, garantizando la seguridad del paciente y procedimientos odontológicos de mayor calidad.

La atención estomatológica del paciente con alteraciones sistémicas y conductuales recae en el especialista estomatólogo en manejo de pacientes sistémicamente comprometidos y con discapacidad, que tenga experiencia en individualización del tratamiento para cada caso en particular y entrenamiento competente del trabajo bajo AG.

El objetivo de este artículo fue presentar el tratamiento odontológico de forma integral (TOI) bajo anestesia general, de un paciente con epilepsia asociado a Discapacidad intelectual moderada, atendido por el servicio de Estomatología de Pacientes Especiales de la Facultad Estomatología de la Universidad Peruana Cayetano Heredia.

\section{Caso clínico}

Paciente masculino de 27 años, procedente del distrito de Comas, Lima-Perú. Acude en compañía de sus padres al servicio de Estomatología de Pacientes Especiales (EPE) de la Clínica Estomatológica Central de la Universidad Peruana Cayetano Heredia. Indican como motivo de consulta dental la presencia de lesiones cariosas, dolor a la masticación y disminución de la ingesta de alimentos; con un tiempo de evolución de 15 días aproximadamente. Presenta como antecedentes patológicos: EP de inicio generalizado tipo tónico-clónico, diagnosticado a los 3 meses de edad, actualmente en controles por neurología. Indica última crisis en el 2012 y medicación actual: Carbamazepina $200 \mathrm{mg} 1$ tableta cada 12 horas. También el informante refiere el diagnóstico de DI (grado moderado).

El examen intraoral es limitado por la poca colaboración del paciente, permitiendo observar únicamente la zona anterior de ambos maxilares, en los que se evidencia acúmulos de placa dura y blanda, encías eritematosas y lesiones cariosas. Se presume presencia de lesiones cariosas en sectores posteriores, acúmulo de placa generalizada, presencia de terceros molares; como diagnósticos por confirmar.

Por lo antes expuesto, el paciente es considerado de alta complejidad para el manejo en el sillón dental, haciéndolo candidato para recibir el TOI bajo AG y de esta manera, poder garantizar el tratamiento de manera segura, eficaz y rápida.

Se explica al familiar el tipo de tratamiento odontológico a realizarse bajo AG y se solicita exámenes auxiliares e interconsultas preoperatorios básicos según el protocolo de nuestra institución para atención ambulatoria bajo AG (tabla 1). A la evaluación de los exámenes auxiliares e interconsultas evidenciamos un buen estado de salud general, estado hemodinámico estable y el dosaje en sangre del anticonvulsivo en rangos terapéuticos adecuados, para la prevención de $\mathrm{CE}$ en el perioperatorio. El día del procedimiento en sala de operaciones (SOP), los cuidadores siguieron las indicaciones brindadas por los médicos (tabla 2) y los estomatólogos; las radiografías periapicales de descarte se realizaron IntraSOP.

El ingreso del paciente a SOP se realizó conjuntamente al personal de anestesiología, 15 minutos antes se administró la premedicación anestésica. Bajo los efectos sedantes se transporta al paciente en silla de ruedas a SOP. Posteriormente, por la falta de colaboración se realizó la inducción anestésica vía inhalatoria inmediatamente seguida de otros medicamentos inductores. Finalmente, el mantenimiento anestésico fue balanceado usando las vías endovenosa e inhalatoria. 
Tabla 1. Exámenes e interconsultas requeridas para la sala de operaciones de este reporte de caso.

\begin{tabular}{|c|c|c|c|}
\hline \multirow[t]{2}{*}{$\begin{array}{l}\text { Tiempo opera- } \\
\text { torio }\end{array}$} & \multicolumn{2}{|c|}{ Exámenes } & Resultados \\
\hline & \multicolumn{3}{|c|}{ Exámenes de laboratorio } \\
\hline & \multicolumn{2}{|c|}{ - Hemograma completo y diferenciado } & Rangos normales \\
\hline & \multicolumn{2}{|c|}{$\begin{array}{l}\text { - Perfil de coagulación (TP, TTP, tiempo de } \\
\text { sangría, tiempo de coagulación, fibrinógeno) }\end{array}$} & Rangos normales \\
\hline & \multicolumn{2}{|c|}{ - Glucosa, urea, creatinina en sangre } & Rangos normales \\
\hline & \multicolumn{2}{|c|}{ - Examen completo de orina } & Rangos normales \\
\hline & \multicolumn{2}{|c|}{ - Dosaje de carbamazepina } & Rangos terapéuticos \\
\hline & \multicolumn{2}{|c|}{ - Transaminasas (ALT y AST) } & Rangos normales \\
\hline & \multicolumn{3}{|c|}{ Interconsultas } \\
\hline & \multicolumn{2}{|l|}{ - Neurología } & $\begin{array}{l}\text { Dosis adecuada carbamazepina para } \\
\text { procedimiento en SOP }\end{array}$ \\
\hline & \multicolumn{2}{|l|}{ - Medicina interna } & Adecuada salud general para procedimiento \\
\hline Preoperatorios & \multicolumn{2}{|l|}{ - Cardiología } & Riesgo quirúrgico I \\
\hline & \multicolumn{2}{|l|}{ - Anestesiología } & Evaluación final y pase para SOP \\
\hline & \multicolumn{2}{|c|}{ - Cirugía y Medicina Maxilofacial } & Atentos al llamado en caso de ser necesario \\
\hline & \multicolumn{3}{|c|}{ Imaginología } \\
\hline Intraoperatorio & \multicolumn{2}{|c|}{ Radiografías periapicales } & Presencia de terceros molares: $1.8,2.8,3.8,4.8$ \\
\hline \multicolumn{2}{|c|}{ Previas } & Del día de SOP & Posteriores \\
\hline \multicolumn{2}{|c|}{ - Ayuno de 8 horas antes } & $\begin{array}{l}\text {-Dosis de medicación: } \\
\text { carbamazepina } 2 \frac{1}{2} \text { tabletas }\end{array}$ & $\begin{array}{l}\text {-Mantener medicación habitual } \\
\text { después de SOP }\end{array}$ \\
\hline \multicolumn{2}{|c|}{$\begin{array}{l}\text {-Preparación de alimentación fría y } \\
\text { blanda necesaria al regresar a casa. }\end{array}$} & $\begin{array}{l}\text {-Vestir ropa cómoda y de fácil } \\
\text { extracción. }\end{array}$ & $\begin{array}{l}\text {-Cumplir con esquemas enviados de } \\
\text { antibióticos y analgésicos }\end{array}$ \\
\hline $\begin{array}{l}\text {-Aseo minuc } \\
\text { noche antes } d\end{array}$ & $\begin{array}{l}\text { so del paciente la } \\
\text { procedimiento. }\end{array}$ & $\begin{array}{l}\text {-Uso de pañal acorde al tamaño del } \\
\text { paciente }\end{array}$ & -Reposo por 48 hrs \\
\hline
\end{tabular}

Bajo los efectos de la AG y entubado por vía nasal, se procede a realizar la evaluación clínica, la toma de radiografías periapicales necesarias (figura 2), set fotográfico de control (figura 3), la confirmación de los diagnósticos y reconfirmación del plan de tratamiento. El plan de tratamiento incluyó: la eliminación del tártaro dental y pulido de las piezas dentarias, obturaciones de resinas y la eliminación de las terceras molares de los cuatro cuadrantes. Se consideró la permanencia de la pieza supernumeraria ubicada entre las piezas 2.2 y 2.3 , por encontrarse en el arco dental sin alterar la oclusión. Se instala el tapón faríngeo, continuando así con el inicio el tiempo operatorio de 2 horas y 30 minutos. Durante la AG el paciente no presento alteraciones en su frecuencia cardiaca ni respiratoria u algún reporte de interés por parte del personal de anestesiología. 


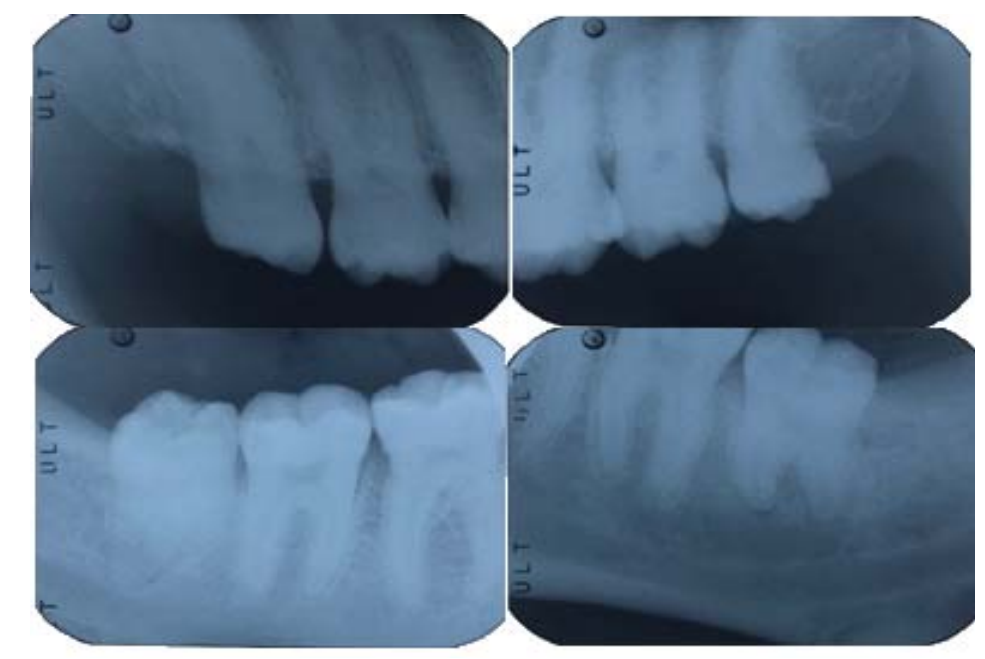

Figura 2. Radiografías periapicales de terceros molares tomadas intraSOP.

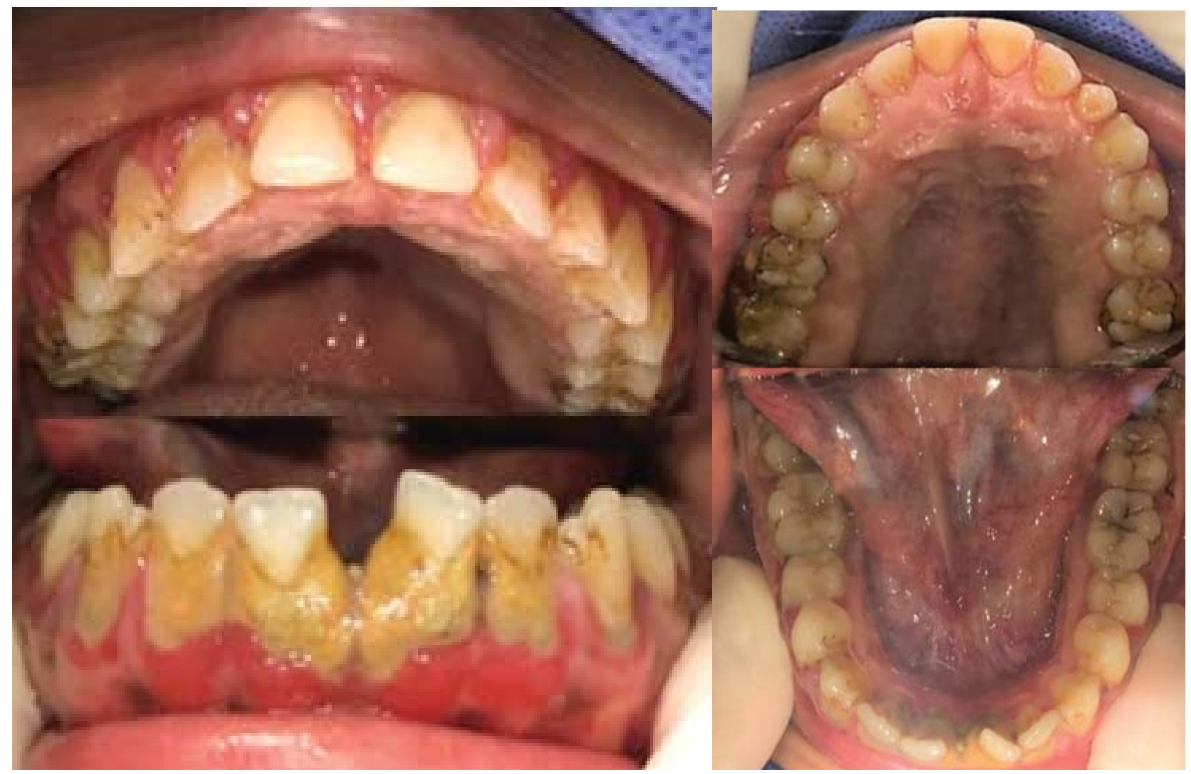

Figura 3. Fotografías intraorales tomadas previo previo al inicio de procedimientos curativos (o tratamiento odontológicos) en sala de operaciones.

Al finalizar los procedimientos en SOP se retiró el tapón faríngeo, extubación y posterior traslado a sala de recuperación, donde se permitió el acceso a un familiar para lograr la tranquilidad del paciente. Se indica medicación ketorolaco $15 \mathrm{mg}$ cada 8 horas por 5 días y clindamicina $300 \mathrm{mg}$, 1 tableta cada 8 horas por 7 días. Se sugiere iniciar con analgésico 6 horas después del egreso de SOP como dosis de rescate y posteriormente continuar con dosis indicadas. Se realizó un control postoperatorio a las 24 horas, sin presencia de complicaciones y otro control 7 días posteriores, donde se aplicó flúor barniz realizando manejo de conducta.

Para este momento se evidencia el cambio evidente en el periodonto, la coloración de la gingiva se presenta rosa, sin apariencia edematosa ni sangrado al contacto y un adecuado proceso de cicatrización de las zonas retromolares, además de aumento en su apetito y estado de ánimo en casa referido por los padres. 
Se brindan instrucciones de higiene oral a los cuidadores y familiares, programándose controles cada 2 meses priorizando la prevención, con el objetivo de evitar en el futuro, que el paciente vuelva a requerir procedimientos bajo AG en SOP (tabla 3 y figura 4).

Tabla 3. Controles y tratamientos postoperatorios en un paciente recurrente o inconstante

\begin{tabular}{|c|c|c|}
\hline $\begin{array}{l}\text { Tiempo } \\
\text { postoperatorio }\end{array}$ & Paciente recurrente & Paciente inconstante \\
\hline 1 día & Control de cirugía y posibles complicaciones & Control de cirugía y posibles complicaciones \\
\hline 1 semana & Control PO, IHO y CBF & Control PO, IHO y CBF \\
\hline $1 \mathrm{mes}$ & Control PO, IHO, profilaxis oral & Control PO, IHO, profilaxis oral y CBF \\
\hline 2 meses & Control PO, IHO, profilaxis oral y CBF & Control PO, IHO, profilaxis oral y CBF \\
\hline 6 meses & $\mathrm{IHO}$, profilaxis oral y CBF & $\mathrm{IHO}$, profilaxis oral y $\mathrm{CBF}$ \\
\hline 1 años & Control PO, IHO, profilaxis oral, $\mathrm{CBF}$ & $\mathrm{IHO}$, profilaxis oral y $\mathrm{CBF}$ \\
\hline \multirow{6}{*}{2 años } & * PLC: manejo de conducta, TRA/resina & *PLC: manejo de conducta, TRA/resina \\
\hline & \multirow{5}{*}{$\begin{array}{l}\text { Control PO, IHO, profilaxis oral, CBF } \\
\text { * PLC: manejo de conducta, TRA/resina }\end{array}$} & $\begin{array}{l}\text { *Evaluación de condición oral general y posible } \\
\text { reingreso a SOP en caso de necesidad }\end{array}$ \\
\hline & & *Evaluación de condición oral general y posible \\
\hline & & \\
\hline & & *PLC: manejo de conducta, TRA/resina \\
\hline & & $\mathrm{IHO}$, profilaxis oral y $\mathrm{CBF}$ \\
\hline
\end{tabular}

Paciente inconstante: Paciente no se presenta a sus citas anteriormente programadas.

PO: postoperatorio, IHO: Instrucciones de higiene oral al familiar/cuidador, CBF: colocación de barniz de flúor (22 600ppm), PLC: presencia lesión cariosa, TRA: Tratamiento restaurador atraumático.

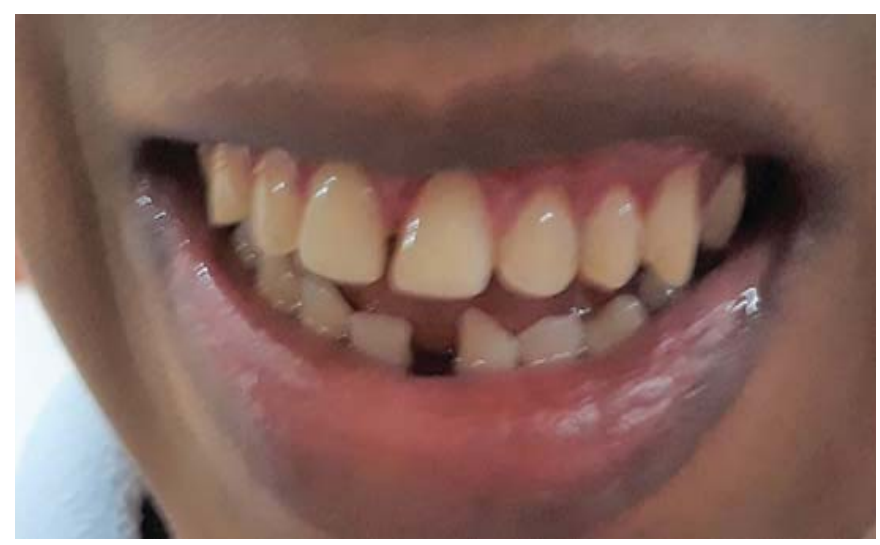

Figura 4. Control postoperatorio a los 7 días. 


\section{DISCUSIÓN}

La elección del trabajo odontológico bajo AG para este paciente fue resultado de una evaluación cuidadosa y completa del caso, ya que cumplía con los criterios para el trabajo bajo esta modalidad. El cumplimiento del protocolo fue completo y la ejecución del TOI permitió realizar los procedimientos odontológicos en una sola intervención, de manera segura, eficiente y de mayor calidad. Así mismo, la planificación de controles dentales programados buscará lograr la colaboración del paciente dentro de lo posible y asegurar la preservación de la salud oral en el tiempo.

Ohtawa et al., mencionan que los principales diagnósticos sistémicos en los que se realiza tratamientos odontológicos bajo AG se asocian con EP $17 \%$, anomalías cardiacas en un $16 \%$, retraso global del desarrollo 50\% (13); también Wolffy et al., indican que la $A G$ está indicada en pacientes con diversas condiciones médicas, físicas o psicológicas que son incapaces de someterse a los tratamientos ambulatorios (14). Sugieren que la EP y DI son indicaciones para el uso de $\mathrm{AG}$, por otro lado, estos casos podrían ser atendidos bajo sedación, lo que afirmamos si es que han fracasado las técnicas de manejo de la conducta en el sillón dental o porque el caso lo requiera.

Por otro lado, al igual que Castillo et al., menciónanos que el trabajo bajo AG permite realizar diagnósticos integrales, en casos que no se puede realizar con anterioridad la adecuada valoración por la falta de cooperación de algunos pacientes (15), por consiguiente, dar una atención integral requerida por el paciente. En tal sentido, coincidimos que el protocolo prequirúrgico para el trabajo bajo $\mathrm{AG}$ es de estricto cumplimiento y este se ha adoptado para una minuciosa valoración médica y estomatológica que busca disminuir el riesgo intra y posoperatorio.

En el reporte de caso de Gutiérrez et al., se afirma que el tratamiento odontológico bajo $\mathrm{AG}$ tiene un riesgo igual que cualquier intervención bajo AG (16). La incidencia de muertes por anestesia oscila entre $1 / 185,000$ a $1 / 300,000$ casos, por lo que podríamos afirmar basados en nuestra experiencia institucional, que el trabajo bajo AG es relativamente seguro. Para llevar a cabo procedimientos de restricción física, sedación o AG, los padres deben tener una clara comprensión de las bondades y beneficios de estos modos de trabajo. No obstante, esta forma de trabajo requiere de un consentimiento firmado por el familiar o apoderado responsable y de una preparación cuidadosa con evaluación previa del balance de riesgos y beneficios $(17,18)$.

Certificamos al igual que Hutchinson, que la intubación bucal es de primera elección para los anestesiólogos por ser una vía más accesible, sin embargo, es probable que este acceso haga difícil el trabajo para el estomatólogo, por otro lado, la intubación nasal en caso de poderse realizar es el método ideal para facilitar el trabajo del odontólogo y preservar intacto el tubo de respiración (17). El uso de gasa como tapón retrofaríngeo es obligatorio, en este paso el cirujano insertará un tampón de gasa en la orofaringe, con el fin de evitar que los líquidos de la pieza de mano, sustancias, fragmentos de dientes u otros puedan introducirse por el esófago y/o tráquea en la cirugía o también sean deglutidos o aspirados durante la fase de recuperación; contrariamente es una conocida fuente de dolor de garganta en el postoperatorio.

Morales et al, en su revisión indica que los pacientes con EP son altamente propensos a presentar manifestaciones en cavidad bucal, en comparación a pacientes no epilépticos, como consecuencia de la mala higiene bucal e inadecuada condición dental (19). Dentro de las lesiones más frecuentes en la cavidad bucal están: caries dental, enfermedad periodontal, pérdida de piezas dentarias, tendencia al sangrado bucal y agrandamiento gingival. Así mismo, las pérdidas dentales por fractura son muy prevalentes en esta población y más aún si, el paciente presenta crisis generalizadas.

El tratamiento odontológico bajo AG según Garcés et al., puede ser conservador o radical y se debe elegir de forma independiente al pronóstico de vida del paciente (20). El tratamiento dental conservador es de elección únicamente cuando se puede asegurar que los procedimientos dentales tendrán pronósticos favorables al $100 \%$. Caso contrario, el tratamiento dental radical se considerará ante la posibilidad del fracaso del tratamiento estomatológico conservador, que tendría consecuencias como: falla en los procedimientos odontológicos, complicaciones de la enfermedad de fondo y/o legales, que demandaría 
nuevamente la entrada a SOP bajo AG para solucionar los inconvenientes producidos por la mala planificación o análisis en la elección de los tratamientos. Coincidimos con los autores que los tratamientos estarán limitados solo a aquellos que se puedan finalizar en una sesión no mayor de 3 horas de tratamientos dentales bajo AG.

Creemos tal como lo mencionan autores como Yeung et al., (5) y Hutchinson (17) que una vez en sala de recuperación, es importante que uno o ambos padres/ cuidadores estén presentes para lograr un ambiente familiar, buscando una cierta tranquilidad del paciente al encontrarse en un lugar desconocido.

El manejo odontológico posterior al procedimiento en SOP es esencialmente preventivo, buscará lograr la adecuación de la conducta para la atención en el sillón dental y entrenar a cuidadores para el mantenimiento adecuado de la higiene oral en casa, de esta manera el objetivo final será resguardar en el tiempo la salud del órgano bucal. Es esencial, mantener un control recurrente del paciente, idealmente cada 2 meses, para lograr evaluar la higiene oral y factores de riesgo. La finalidad será evitar la aparición de enfermedades bucodentales que requiera de otra intervención en SOP (tabla 3).

\section{CONCLUSIONES}

Con base en la literatura consultada, se concluye que los pacientes con EP asociada a DI son altamente propensos a presentar lesiones y alteraciones en la cavidad bucal. Requieren atención y evaluación estomatológica constante que, en algunos casos, no puede realizarse en el sillón dental, siendo necesario el TOI bajo AG. El protocolo para el trabajo bajo AG debe incluir las respectivas interconsultas, exámenes de laboratorio y auxiliares que permitan conocer el estado sistémico del paciente. Establecer adecuados diagnósticos sistémicos logra minimizar la posibilidad de complicaciones durante el tiempo operatorio.

El tratamiento odontológico en este paciente fue exitoso y oportuno, el mismo debe ser apoyado con el cumplimiento de los controles periódicos y el mejoramiento de la higiene oral diariamente por sus cuidadores.

\section{Correspondencia}

Ana Cristina López,

Correo electrónico: ana.lopez@upch.pe

\section{REFERENCIAS BIBLIOGRAFICAS}

1. Managing patients with special needs. Dent Abstr. 2014;59(3):e61-2.

2. Sandín B. DSM-5: ¿Cambio de paradigma en la clasificación de los trastornos mentales? Rev Psicopatología Psicol Clínica. 2013;18(3):255-286.

3. Mielnik M, Skawińska A, Michałowski A, Błaszczak J. Problems with access to dental treatment for children with epilepsy. Epilepsy Behav. 2018;81:107-10.

4. Organización Mundial de la Salud. Epilepsia. Un imperativo de la salud pública. Suiza; Organización Mundial de la Salud; 2019. (Citado el 15 de setiembre del 2020) Disponible en: www.who.int/mental health/ neurology/epilepsy/report_2019/en

5. Morgan H, Abou El Fadl R, Kabil N, Elagouza I. Assessment of oral health status of children with epilepsy: A retrospective cohort study. Int J Paediatr Dent. 2019;29(1):79-85

6. Lisowska P, Daly B. Vagus nerve stimulation therapy (VNST) in epilepsy - implications for dental practice. Br Dent J. 2012;212(2):69-72.

7. Fisher R, Acevedo C, Arzimanoglou A, et al. ILAE Official Report: A practical clinical definition of epilepsy. Epilepsia. 2014;55(4):475-82.

8. Prasad AN, Prasad C. Genetic Aspects of Human Epilepsy. En: Rimoin D, Pyeritz R, Korf B. Editores. Emery and Rimoin's Principles and Practice of Medical Genetics. New York: Elsevier; 2013.p. 1-40. (Citado el 15 de setiembre del 2020) Disponible en: https://linkinghub.elsevier.com/retrieve/pii/ B9780123838346001221

9. Fisher R, Cross J, French J, et al. Operational classification of seizure types by the International League Against Epilepsy: Position Paper of the ILAE Commission for Classification and Terminology. Epilepsia. 2017;58(4):522-30.

10. Scheffer I, Berkovic S, Capovilla G, et al. Clasificación de las epilepsias de la ILAE: Documento de posición de la Comisión de Clasificación y Terminología de la ILAE. Epilepsia. 2017;58(4):512-21.

11. Schöpper M, Ludolph A, Fauser S. Dental care in patients with epilepsy: a survey of 82 patients and their attending dentists and neurologists in southern Germany. Int Dent J. 2016;66(6):366-74.

12. Yeung P, Wong V, McGrath C, Yiu C, Lee G. Oral health status of children with epilepsy in Hong Kong. J Investig Clin Dent. 2019;10(4). doi: doi/abs/10.1111/ jicd.12479

13. Ohtawa Y, Yoshida M, Fukuda K. Parental Satisfaction 
with Ambulatory Anesthesia during Dental Treatment for Disabled Individuals and Their Preference for Same in Future. Bull Tokyo Dent Coll. 2019;60(1):53-60.

14. Wolff A, Singer A, Shlomi B. Comprehensive dental treatment under general anaesthesia. Dent Cadmos. 2014;82(3):182-8.

15. Castillo MR, Perona MPG, Kanashiro IC, Perea PM, Silva- Esteves RF. Estomatología Pediátrica. Madrid: Ripano Editorial Médica; 2010.

16. Gutierrez L, Cardenas M, Guadarrama L. La odontopediatría como parte del equipo multidisciplinario hospitalario durante la rehabilitación bucal bajo anestesia general. Reporte de tres casos. Arch Inv Mat Inf. 2014;6(2):67-74.

17. Hutchinson S. General anaesthesia for dentistry. Anaesth Intensive Care Med. 2014;15(8):366-9.

18. Luengas M, Luegas E, Sáenz L. Atención odontológica a personas con discapacidad intelectual: una cuestión de derecho. Rev ADM. 2017;74 (5): 269-274:6.

19. Morales A, Hallal Y, Quintero F, Rondón I. Epilepsia y sus implicaciones en el campo odontológico: Artículo de Revisión. Acta Bioclínica. 2014;(4):34-59.

20. Garcés A, Cárdenas M, Robles N, Guadarrama L. Rehabilitación bucal bajo anestesia general en pacientes pediátricos del Centro de Especialidades Odontológicas. Arch Inv Mat Inf. 2012;4(2):63-70.

Recibido: 15-04-19

Aceptado: 20-09-20 